\title{
Towards Data-Driven Evaluation of Site Effects From Coda of Strong Motion Timeseries Using Recurrent Neural Networks
}

Mona Izadi ( $\sim$ mona.izadi@sds.dpri.kyoto-u.ac.jp )

Kyoto University https://orcid.org/0000-0003-3321-3785

Shinichi Matsushima

Kyoto University

Full paper

Keywords: Coda waves, Site effects, Fourier analysis, Neural networks, Time-series analysis, Computational seismology, Earthquake ground motions

Posted Date: November 12th, 2021

DOI: https://doi.org/10.21203/rs.3.rs-986573/v1

License: (c) (1) This work is licensed under a Creative Commons Attribution 4.0 International License.

Read Full License 


\section{Title page:}

2 Title: Towards data-driven evaluation of site effects from coda of strong motion timeseries

3 using recurrent neural networks

4 Author \#1: Mona Izadi, Ph.D Candidate, Graduate School of Engineering, Kyoto University, Japan,

$5 \quad$ mona.izadi@sds.dpri.kyoto-u.ac.jp

6 Author \#2: Shinichi Matsushima, Professor, Disaster Prevention Research Institute, Kyoto

$7 \quad$ University, Japan, matsushima@sds.dpri.kyoto-u.ac.jp

8

9 Corresponding author:

10 Mona Izadi

11 Graduate School of Engineering, Kyoto University, Japan

12 Phone: +81-774-38-4076

13 Fax: +81-774-38-4075

14 E-mail: mona.izadi@sds.dpri.kyoto-u.ac.jp 


\section{Abstract}

18 It is known that coda of strong motion records are products of numerous scatterings of body and

19 surface waves within the subsurface soil structure. Several studies have successfully simulated coda wave envelopes by modelling the energy decay. However, due to limitations of quantifying soil heterogeneities, deterministic simulation of scattered wavefields is much more challenging. Therefore, the reverse problem of estimating non statistical properties of subsurface structure from coda waves remains a theoretical potential. On the other hand, machine learning techniques have proven useful in dealing with problems of similar nature, where a theoretical solution is imaginable yet hard to achieve due to a great number of unknown variables. This study utilizes artificial neural networks to propose a new approach of evaluating site effects from coda waves, with the future prospect of obtaining the similar results from microtremor records. A Long ShortTerm Memory recurrent neural network is designed using Tensorflow 2 library in Python language. The study utilizes a strong motion dataset consisting of about 60000 3-component records as well as borehole data at 464 stations of Kiban-Kyoshin Network across Japan. The prediction input is coda wave timeseries of strong motion records, defined based on a parametric 
sequential features. In the first step, the prediction target is a vector of 3 site effect proxies namely,

34 time-averaged shear-wave velocities for the upper 30-m depth Vs30 and the upper 10-m depth

$35 \mathrm{Vs} 10$ and predominant frequency $f_{0}$. In this step, different model parameter combinations are

36 tested to ensure the basic model's ability in extracting site-specific information from the input

37 coda waves. One of the combinations is then used in the second step, in which the prediction

38 target is surface to downhole ratio of Fourier Amplitude Spectra. For each of the 3 components

39 EW, NS and UD, 100 identical networks are trained to each predict the desired ratio at a certain

40 target frequency. Accuracy of test sample predictions confirms applicability of the proposed

41 approach as well as its potential for future works on microtremor timeseries instead of coda waves.

\section{Keywords}

43 Coda waves, Site effects, Fourier analysis, Neural networks, Time-series analysis, Computational

44 seismology, Earthquake ground motions

\section{Main Text}

\section{$46 \quad$ Introduction}

47 In the recent years, machine learning (ML) techniques, especially multilayer Artificial Neural

48 Networks (ANNs) have gained growing attention in all fields of science and engineering. Due to 
49 great advances in the flexibility and performance of algorithms as well as programming and

50 computational efficiency, machine learning techniques are already in use for many real-life

51 applications such as medical diagnosis or engineering defect detection. However, one of the fields

52 in which machine learning remains to be limited to research level is seismological and earthquake

53 engineering.

54 From physics-based ground motion prediction to building response analysis and deterministic

55 seismic hazard analysis, a wide variety of seismological procedures demand an accurate

56 estimation of the site effect as a crucial analytical component. Traditionally, the site effect is

57 accounted for via either averaged parameters such as peak ground acceleration (PGA) and time-

58 averaged shear wave velocity to $30 \mathrm{~m}$ depth (Vs30) or spectral evaluations based on

59 deconvolution of waveforms.

60 Both above methods have their respective demerits when it comes to engineering applicability.

61 For instance, single parameter evaluation neglects time-dependent and/or frequency-dependent

characteristics and spectral evaluations still contain uncertainties regarding directionality as well

as nonlinear behavior of subsurface structure. 
64 Considering the availability of a great number of strong motion records, it is not inconceivable that there may be an advantage in adopting ML algorithms such as ANNs to better understand and evaluate site effects and directly extract as much information as possible from past recorded data. Furthermore, as opposed to full waves, the coda of strong motion records is known to contain fully scattered body and surface waves, which makes them an immediate candidate for extraction of site-specific information.

A review of literature by Mignan et al. (2019) reveals that ANNs have been increasingly adopted in seismological studies in a range of topics such as ground motion prediction, aftershock prediction and seismic waveform analysis. Although, in some cases, the results seem satisfactory compared to conventional predictive methods, for example, prediction of aftershock patterns by

DeVries et al. (2018) or large earthquake magnitudes by Huang et al. (2018), it seems there is not enough justification yet to adopt the proposed methods in engineering or risk assessment practice. Therefore, besides obtaining satisfactory results, a persistent challenge regarding the use of ML methods is to validate the formulation by theoretical explanation.

In this research, strong motion datasets are used to train an ANN to ultimately estimate a spectral 
80 Kiban Kyoshin Network (KiK-net) stations operated and maintained by the National Research

81 Institute for Earth Science and Disaster Resilience (NIED) (Aoi et al., 2004) are obtained and a

82 Recurrent Neural Network (RNN) with Long Short-Term Memory (LSTM) cells is trained on the

83 coda window of time-series data to predict site effect proxies and site amplification ratios for each

84 station. In the following sections, after the introduction of utilized data and method, analysis

85 approach and results are discussed, followed by indications regarding applicability of the method

86 as well as physical interpretation of the results.

87 It is worth noting that the future prospect of this study is to train networks on available coda waves

88 but make predictions from observed microtremor timeseries on sites where earthquake records

89 are not available. This expectation is based on observed evidence of fully scattered waves and energy equipartition in both microtremors and coda of strong motions, indicating that both may reflect similar information on subsurface structure at specific sites. Indications of the current stage of the study in relation to this prospect are also discussed in following sections.

\section{$94 \quad$ Data processing}


96 Strong motion seismographs are used in this study for three purposes. Firstly, for extraction of a

97 large number of coda waves, secondly, for computation of earthquake horizontal-to-vertical

$98(\mathrm{H} / \mathrm{V})$ spectral ratios (EHVRs) and predominant frequencies at each site and lastly, for

99 computation of site amplification ratios for different earthquake events at different sites.

100 The initially selected dataset contains EW, NS and UD components of strong motion acceleration

101 timeseries with PGAs below $100 \mathrm{~cm} / \mathrm{s}^{2}$ and above $10 \mathrm{~cm} / \mathrm{s}^{2}$, recorded at all KiK-net stations

102 between 2000 January first and 2020 January first (Aoi et al., 2004; NIED, 2019). Records with

103 PGAs below $10 \mathrm{~cm} / \mathrm{s}^{2}$ tend to have short durations, making it difficult to detect coda time windows

104 and thus were not considered for convenience.

105 The original sampling rate of all the timeseries data is $100 \mathrm{~Hz}$. Because the RNN algorithm

106 performs better with shorter sequences, all timeseries had to be resampled at the smallest

frequency possible. Considering that the frequency range of engineering interest is generally up

to $10 \mathrm{~Hz}$, without losing any information in this range, the smallest Nyquist frequency would be

$10 \mathrm{~Hz}$. Therefore, all timeseries used in this study were resampled at $20 \mathrm{~Hz}$. Next, in order to

Butterworth filter. 
112 In order to ensure that only records within the linear soil response range are included, the higher

113 limit of PGA was adjusted per station, based on the variance of predominant frequencies. First,

114 the predominant frequency $\left(f_{0}\right)$ was calculated from the EHVR of each strong motion record. At

115 each site, mean and standard deviation (SD) values of the estimated predominant frequencies

116 were computed. Those records with $f_{0}$ within the mean $+/$ - SD range were kept as linear samples

117 and those with exceeding values were eliminated.

119 Site effect proxies

120 Initially five site proxies were calculated for each station, Vs30 and time-averaged shear-wave

121 velocities for the upper 10-m depth (Vs10), depth of engineering bedrock $\left(D_{\mathrm{b}}\right)$, surface to bedrock

ratio of S-wave velocity $\left(R_{\mathrm{v}}\right)$ and $f_{0}$. However, when multiple trial networks were trained on the

dataset and all showed poor performances for two of the proxies, namely $D_{\mathrm{b}}$ and $R_{\mathrm{v}}$, the two were

removed from further investigations. The final site effect proxies' vector for each station was built

from the remaining 3 proxies, which are Vs30, Vs10 and $f_{0}$.

$f_{0}$ at each station was calculated as the first peak frequency of average EHVRs of all earthquake 
smoothed using a Parzen window with bandwidth of $0.1 \mathrm{~Hz}$. The prominent peaks were then

selected as all local maxima points where peak prominence is larger than 1, excluding frequencies that are smaller than $0.1 \mathrm{~Hz}$ and thus outside the engineering interest range. In this case, peak prominence is defined as the vertical distance between a peak point and its lowest contour line. Fig. 1 shows examples of EHVR plots and selected predominant frequencies at six KiK-net stations.

As seen in the figure, in cases like MIEH05 where no peaks in the lower frequency range pass the criteria, inevitably, $f_{0}$ is detected in higher frequency range. This means that the same site proxy or RNN feature contains certain information (lower frequency content) at some data points and different information (higher frequency content) at others. Although this inconsistency in a feature vector is far from optimal for training a neural network, any proper solution would have compromised fast and automatic calculation of the proxy values. Furthermore, estimation of practical proxies is not the main goal in the first step of this study and so the issue was considered negligible. 
144 A known characteristic of coda waves is decaying energy content. Since it was first discussed by

145 Aki (1969), several studies have formulated the theory behind this behavior, through various

146 models such as multiple scattering or radiative transfer function. Utilizing a dense array of

147 seismographs, Shapiro et al. (2000) have shown that while deformation energy continuously

148 decays following the S-wave arrival, shear to compressional energy ratio stabilizes for a while

149 before largely fluctuating again. Their findings were in agreement with diffuse wave field theory,

150 indicating energy is equipartitioned during the coda window. Using this same method, Margerin

151 et al. (2009) have observed equipartition in coda waves and successfully applied it to model a

152 layered media in Pinyon Flats. Although this method is promising, it relies on accurate estimation

153 of divergence and curl of the wave field through observations of a dense array. Therefore, in order

154 to determine the coda time window from single station records in this study, H/V energy ratio

was considered instead, as an indicator of equipartition. Energy envelopes were computed for

each seismograph by Eq. (1) following Mayeda \& Walter (1996):

157

$$
e n v(t)=\sqrt{\left(v^{2}(t)+h^{2}(t)\right)}
$$




$$
r_{\text {mean }}=\operatorname{mean}\left(\frac{\int_{t_{1}}^{t_{1}+5} e n v_{\text {smooth_h }}(t)}{\int_{t_{1}}^{t_{1}+5} e n v_{\text {Smooth_v }}(t)}\right)<2
$$

in which $e n v_{\text {smooth }}(t)$ is the envelope function smoothed by a Hanning window of 10 s width.

Since the parameters are evaluated only in relation to threshold values, the smoothing window

width has negligible effect on the outcome. $e n v_{\text {smooth_h }}(t)$ and $e n v_{\text {smooth_v }}(t)$ refer to average energy envelope of the two horizontal components and energy envelope of the vertical component, respectively. Fig. 2 shows an example of applying this criterion to EW components of a M3.7 event (sample record 1) and a M6.5 event (sample record 2) observed at the surface of MIEH05 station in Mie prefecture. As seen in Fig. 2b, the H/V energy ratio in a lot of records does not show a clear decline. In such cases, this step is not applicable and the initial onset $t_{1}$ is set as 0 . 
174 Since samples with varying PGA values exhibit varying energy compositions of S-wave time-

175 window, relying on the decline of H/V energy ratio is not enough to detect a coda onset. To create

176 a unified parameterized criterion for all samples, the ratio of energy prior to coda onset to the total

177 energy of the seismograph (ER) was defined as a control parameter. Based on this parameter, an

178 alternative onset was defined as the timestep $t$ where $\operatorname{er}(t)$ drops to a certain value (e.g.,

$\left.179 e r_{\text {thres }}=0.8\right)$. Between the initial and alternative onsets computed for each sample, the later one

180 of the two was chosen as final onset to avoid high amplitudes as much as possible. On the other

181 hand, in order to determine the end of coda, coefficient of variation (CV) for remaining portion

182 of seismograph following coda was used as a control parameter and the end of coda was

183 determined as the timestamp $t$ where $c v(t)$ reaches a certain lower limit (e.g., $c v_{\text {thres }}=0.05$ ).

184 The following two equations sum up this procedure.

185

$$
\begin{gathered}
c v(t)=\frac{\operatorname{std}\left(e n v_{\text {smooth }}(t)\right)}{\operatorname{mean}\left(e n v_{\text {smooth }}(t)\right)} \leq c v_{\text {thres }} \\
\operatorname{er}(t)=\frac{\int_{0}^{t} e n v_{\text {smooth }}(t)}{\int_{0}^{\infty} e n v_{\text {smooth }}(t)} \geq e r_{\text {thres }}
\end{gathered}
$$


187 The values of $e r_{\text {thres }}$ and $c v_{\text {thres }}$ can be optimized during the RNN training, for highest 188 prediction accuracy. Figs. 3 and 4 show examples of applying this coda extraction criteria to 189 sample records 1 and 2 of Fig. 2, respectively. In each figure, the top row shows the extracted 190 coda time-window with the criteria $e r_{\text {thres }}=0.8$ and $c v_{\text {thres }}=0.1(\mathrm{C} 1)$, and the bottom row 191 shows the extracted coda time-window with the criteria $e r_{\text {thres }}=0.9$ and $c v_{\text {thres }}=0.2(\mathrm{C} 2)$. 192 Compared to C2, C1 picks an earlier onset and a later more stabilized end point.

193 By this method, coda time-windows with different lengths are extracted from each record sample.

194 In order to create fixed length input vectors for training RNNs, only the last 10 seconds of each

195 extracted coda wave was used for extracted time-windows longer than 10 seconds, and 10-second vectors were created by zero padding the vector head for extracted time-windows shorter than 10 seconds. In the end, each sample input consists of three timeseries (EW, NS and UD) with 200 timestamps at a $20 \mathrm{~Hz}$ sampling rate. As RNNs have a better performance on shorter timeseries data, the 10-second length was chosen because it is the minimum duration necessary to contain the lower limit of our frequency range of interest in this paper which is $0.1 \mathrm{~Hz}$. 
standardized, and downhole S-wave velocities differ from station to station. For these reasons,

unlike records at a nearby reference outcrop rock, the downhole records are not explicitly

controlled by just source and path characteristics. In order to account for this discrepancy when

computing site transfer functions based on downhole data, some studies have suggested correction

212 functions for surface to downhole spectral ratios (Héloïse et al., 2012). However, since the

purpose of this study is not to predict the transfer function but to examine the capability of an

RNN model in predicting site effects from coda timeseries data, such procedures were deemed

unnecessary. It was assumed for the purpose of this study that KiK-net downhole records are

ground motion at engineering bedrock, containing main characteristics of earthquake source and

217 propagation path which can be implicitly recognized by a data-driven model. This means that our 
219 local sedimentary layers at each station. Nevertheless, this divergence from ground motion at

220 outcrop is a function of the same shallow subsurface structure which we expect to be represented

221 by the coda waves. Therefore, although this divergence is not explicitly quantified and corrected

222 for each station, the RNN model is expected to implicitly capture and generalize it to all stations,

223 thus learning consistent patterns. If the model is proved to work using downhole data, it can be

224 used on corrected ground motion at engineering bedrock for practical use.

225 In literature, several deterministic methods exist to calculate ground motion at engineering

226 bedrock from appropriate parameters to account for source mechanism as well as path attenuation.

227 An early example is the approach of Boore (1983) which is a stochastic approximation of ground

228 motion envelope based on two most basic parameters of magnitude and distance. Another

example is the method by Satoh (2004) which utilizes inversion of group time delay to improve

230

Boore's envelope approximation and simulate full length time series with much higher precision.

231 Once any such technique is employed, in order to simulate ground motion characteristics at

232 surface level, only site amplification effects remain unaccounted for.

233 In the second step of this study, RNNs are used to predict ground motion Fourier amplitude 
bedrock respectively.

$$
r s b_{\mathrm{FAS}}=\frac{F A S_{\mathrm{s}}(\omega)}{F A S_{\mathrm{b}}(\omega)} \forall \omega \in[0.0,0.1, \cdots, 10.0]
$$

\section{Method}

Modelling sequential data with RNN

Based on the information flow, ANN architectures can be broadly divided into two types, feed- 
each layer serves as the input to the next layer without loops. In contrast, by utilizing an

251 information flow different from feed-forward architectures, recurrent type architectures are

252 intended to account for the order in sequential data. In this case, the input to each hidden layer is

253 not only the previous layer's output, but also the output of the same hidden layer from the previous

254 time stamp. This way, during training, the updating of weights automatically depends on the order

255 of the data. In graph notations, this order-dependency or sequential nature of weights in hidden

256 layers is represented by a loop (Figs 5 and 6), also called a recurrent edge (Raschka \& Mirjalili,

257 2017).

258 The training process of machine learning models requires the definition of an error function as

259 well as an efficient method to minimize the error generated by this function in each training epoch.

260 Since the error minimization involves calculating weight derivatives and due to large weight

261 matrices in ANN models, training these models was long a challenge compared to simpler

262 machine learning techniques. Since its introduction by Rumelhart et al. (1986), back-propagation

263 has been the best solution for this challenge and the most powerful and widely used method of

training feed-forward ANNs. 
However, the same back-propagation techniques do not work as favorably on RNNs. Since in

266 RNNs the hidden layer contains terms from all previous timestamps, its loss computation requires

267 multiplication of many weight values corresponding to adjacent timestamps. This means backpropagation of errors generates weight derivatives powered by large values, especially in RNN models with long range dependencies. Therefore, very large or very small weight derivative values arise the problem of vanishing or exploding gradient.

271 To overcome this challenge several techniques have been introduced, amongst which the use of

272 LSTM networks, introduced by Hochreiter \& Jürgen (1997), is currently most efficient and

273 popular. The building blocks of these networks are LSTM cells, which contain a memory of the

274 cell state upon the introduction of each new sample. These cell states are designed in a way that generate weight matrices with determinant values of nearly 1 to avoid the vanishing or exploding gradient problem. The network is then trained by backpropagating the error to these support matrices and updating them instead of the original weight matrices. 

the output value. The input data is a three-dimensional (3D) tensor standard to all RNNs, containing a sequence of feature vectors for each sample.

283 A sigmoid or tanh activation function is used in order to obtain a continuous predicted value for 284 the site proxies or amplification ratios, against which, the prediction loss and errors of the network are calculated. The target value is the min-max scaled true value of site proxies and amplification ratios. A visual annotation of this network is shown in Fig. 5. and predict site effect proxy or site amplification values of the associated station and each of the three ground motion components EW, NS and UD are treated as independent features. Therefore, the input 3D tensor is designed with dimensions $n_{s} \times 100 \times 3 ; n_{s}$ being the number of training samples, 100 and 3 being the number of timestamps in each input timeseries (sequential sample) and the number of components (sequential features), respectively.

295 A significant factor that affects the performance of machine learning models is the quality of data.

296 Specifically, in problems involving big data of any sort, ensuring this quality becomes more 
While there are many well established methods such as the principal component analysis for accurate detection of non-sequential outlier data points, handling sequential outliers is not as straightforward. The problem of detecting anomalous timeseries in a timeseries dataset is either an unsupervised one, relying only on input data, or a supervised one, employing the target information as well. In both approaches, outlier analysis could be done by either clustering data points via similarity functions or parametric approaches which do not specify anomalous data but instead assign an anomaly score to each data point (Gupta et al., 2013). In the latter approach, a threshold score can be defined as a control parameter to be adjusted and find just the right borderline between good and bad data points.

In this study, the input timeseries are coda waves extracted from earthquake seismographs,

311 through the energy-based procedure explained in the previous section. Among these generated 
include timeseries that passed the coda wave criteria but are dominated by non-scattered body

314 waves or consist of mainly device noise. But even the desired coda waves are supposed to be fully

315 scattered waves and thus noisy data. In this case, clustering algorithms may not be able to

316 precisely map the innate correlation among data points. Therefore, it is preferred to take a

317 parametric approach rather than determining the outliers by strictly modelling correlations.

318 Following a similar method to Malhorta et al. (2016) and Kieu et al. (2018), an autoencoder RNN

319 is utilized as the outlier analysis method in this study. The autoencoder RNN is trained to take an

320 input signal, learn and encode it via LSTM hidden layers and reconstruct and decode it to return

321 the same signal as the output. The anomaly score of each input signal is then defined as its

322 reconstruction error.

323 The autoencoder model is an LSTM network with two recurrent hidden layers each composed of

32472 LSTM cell units, and one feed-forward dense hidden layer. Since an autoencoder is a model

325 to encode the input data and decode it back to the same data, input and output data have the same

326 dimensions, in this case three-dimensional tensors containing three component coda wave

327 timeseries. A visual annotation of this network is shown in Fig. 6. 
Firstly, a coda sample containing three component timeseries is fed into the RNN encoder and the model is trained over 200 epochs to reconstruct the input signal. After the training is complete, dominated, the timeseries should exhibit relatively stationary fluctuations with regular shapes.

334 Therefore, the autoencoder is expected to be more successful and return smaller MSE values when

335 reconstructing the outliers rather than the actual noisy coda waves.

336 To decide the threshold outlier score, microtremor data was used as reference because of its more

337 reliable content of scattered seismic waves. The microtremor used for this purpose is a data set by Kawase et al. (2015). Fig. 7 shows some example comparisons between original and reconstructed signals for three components of random coda timeseries with high and low reconstruction errors, as well as a random microtremor timeseries. It can be confirmed that coda samples with smaller reconstruction errors have long periods and regular fluctuations while those 
344 0.3. All coda samples with lower scores were eliminated. This means that in some stations like

345 EHMH04 of Fig. 7, no coda samples passed the criteria, thus such stations were not represented

346 in the final learning dataset.

347 Satoh et al. (2001) have shown that at soft soil sites the HVRs calculated from earthquake coda

348 are contaminated by Rayleigh waves. Therefore, in frequencies lower than 3, maximum peaks of

349 HVRs for coda waves lie somewhere between those for microtremor and earthquake S-wave. This

350 was observed in our investigations as well. To evaluate the possible effects of outlier analysis on

351 the average properties of our coda dataset, HVRs were compared at 105 stations, where

352 microtremor records were available. Fig. 8 shows examples at three stations, in which bold lines

353 show average HVRs calculated from coda dataset (blue), outlier-removed coda dataset (black),

354 segmented microtremor dataset (red) and selected earthquake dataset (green) and light-colored

355 lines show standard deviations.

356 As evident from the example plots, the elimination of outliers makes no significant difference in

357 EHVRs. It can be concluded that the detected and eliminated outliers are indeed noise dominated

stationary signals and have no apparent relation with site effects or even S-wave content. 
362 This first step of the study, namely prediction of site effect proxies, seeks two main purposes.

363 First, to construct an RNN model and enable it to extract information on site effects directly from

364 coda wave timeseries. Second, to evaluate the similarity between coda waves and microtremor

365 waves regarding the reflection of site-effects in each of the two timeseries. Therefore, a parametric

366 investigation was conducted to find suitable coda wave extraction parameters on one hand, and

367 best model parameters on the other.

368 Coda waves were extracted from strong motion timeseries according to the steps described in the previous section. The parameters $e r_{\text {thres }}$ and $c v_{\text {thres }}$ were given two combinations of values, one with a loose energy criterion $(\mathrm{C} 1)$ in which, $e r_{\text {thres }}=0.8$ and $c v_{\text {thres }}=0.1$, and another with a stricter energy criterion $(\mathrm{C} 2)$ in which $e r_{\text {thres }}=0.9$ and $c v_{\text {thres }}=0.2$.

RNN models were designed with four different parameterized structures as shown in Table 1. The two structure parameters are number of LSTM hidden layers (n_LSTM) and activation function, resulting in four different models. 

computed for test and training sets for all three proxies. The results are summarized in Table 2. For each proxy, the values are color coded from blue to white, for smallest to largest errors and deviations. As expected, training samples derive smaller errors than test samples.

383 For all three proxies, all eight combinations have yielded very similar performances and converged at similar rates, after about 400 epochs. This means that for a better selection of the coda definition criteria, more hyperparameter combinations should be evaluated through a more vigorous tuning method such as Grid Search. Since all combinations examined in this step were equally successful at learning site effect proxies from coda waves, only one combination (model ratios. 
397 The second step of this study attempts to predict site amplification ratios for a given strong motion event at a given site, from an input of three coda timeseries representing site effects. For simplicity, site amplification ratios are defined here as SBRs of FAS. To achieve equal representation of all stations, 40 coda waves were randomly chosen for each station. Because of the varying number of coda waves that passed the $\mathrm{C} 1$ criteria and the outlier analysis at each station, some stations in the dataset had less than 40 coda waves. For these stations, 

components at 100 time or frequency bins (300 target values).

Prior to training the models, the dataset was divided into training and test sets with the ratio of separate cases with different test set definitions were considered. In the first case (Cs from this point on), about 10 percent of stations were defined as test stations and all their coda waves were kept out of training. Results of this test set are to exhibit the networks performance on unseen 413 locations. In the second case (Cc from this point on) 10 percent of the coda waves from each 414 station, meaning four samples, were kept out of training. The results of this test set are to help 415 interpret Cs test results by exhibiting the networks performance on unseen coda samples at seen locations.

Table 3 shows average Mean Absolute Errors (MAEs) and SD of MAEs for test and training sets of both cases, with cells color coded from dark blue to white indicating lowest to highest errors. As expected, Cc test samples derive closer errors to training test samples, confirming that similar to the first step, the network is able to generalize coda samples recorded at the same station to derive same relevant targets, in this step, amplification ratios. 
Fig. 10 shows average MAE and SD of prediction errors per station. Although test set of case Cc

424 in the figure, for the purpose of comparison. The bars are sorted by the difference between Cc

425 and Cs errors divided by the Cs error, in a decreasing order from left to right. The following three

426 observations can be made regarding this figure: 1 . The stations with medium Cs accuracies

427 (medium length bars) are scattered from left to right, showing varying degrees of Cc

428 outperforming Cs. 2. The lower Cs errors are towards the left side, meaning Cc has a much better

429 performance at stations with better accuracies. 3. The biggest Cs errors tend to be towards the

430 right side, meaning the $\mathrm{Cc}$ and $\mathrm{Cs}$ performances do not differ much in stations with lower

431 accuracies.

432 Fig. 11 shows examples of three stations with similar Cs as well as Cc errors. It can be observed that most of these stations do not have distinct peaks and although $\mathrm{Cc}$ in general outperforms $\mathrm{Cs}$, both predictions are almost within the uncertainty range of true values. It shows that in absence of extreme variations, the model trained on current dataset exhibits sufficient predictive accuracy on unseen locations. 
437 Fig. 12 shows examples of three stations with relatively low Cs errors and highest difference

438 between Cc and Cs errors (left hand bars on Figure 10). We can see from Cs figures on top that

439 although the networks can detect the overall trend at new stations, the peaks are not coherent. On

440 the contrary, the Cc case shows much more precision with coherent peaks as well as amplitudes

441 except for the lower frequency peak at AICH06. This shows that only in cases where the model

442 can generalize learnt trends to unseen locations (low Cs error), presence of more closely related

443 data in training set increases coherence of peaks and variation of amplitudes.

444 Fig. 13 shows examples of three stations with highest Cs as well as Cc errors (right hand bars on

445 Fig. 10). Both high and low frequency peaks in these cases have high amplitudes and are vastly

446 underestimated by the model in Cc case. The Cs case also, despite detecting the peaks, equally

447 underestimates the amplitudes. It shows that although the presence of closely related samples in

448 dataset, enables the network to detect coherent peaks, estimation of amplitudes relies on the

449 general trend learnt from all training samples. This is most clear in case of lower frequency peak

450 at MYGH01 which is not well detected by Cs case. Thus, for more accurate results on unseen

451 locations, the presence of representative training data alone is not enough but the number of 
453 These observations can be confirmed with more confidence by examining scatter plots of

454 prediction results at different frequencies. Fig. 14 shows predicted values for training and test sets

455 of both Cs and Cc cases, plotted against true values for three example frequencies $9.8 \mathrm{~Hz}, 4.8 \mathrm{~Hz}$

456 and $0.8 \mathrm{~Hz}$. At each frequency, in middle range amplitudes where training samples are most

457 populated, predictions of Cc test samples are as good as those of Cs test samples. These values

458 correspond to stations without distinct peaks such as example stations in Figure 12. As the

459 amplitudes get larger and training samples sparser, Cs test results deviate from true values faster

460 than Cc test results. At amplitudes larger than about 10 for high and 3 for low frequency ranges,

461 the trend of Cs test sample predictions starts to flatten, but for Cc test samples this does not happen

462 until much larger amplitudes. At these amplitudes, overall pattern of Cs test samples is still

captured but with lower definition than $\mathrm{Cc}$, as previously displayed by examples in Figure 13. On

the other hand, in amplitudes that are much larger than median and training samples are

remarkably sparse, values of both Cs and Cc test samples are equally underestimated. These

amplitudes correspond to underrepresented stations similar to examples in Figure 14.

467 It can be concluded that accurate estimation of amplitudes depends on sufficient representation 
certain amplitude ranges, the network can generalize learnt trends to the same ranges at unseen

470 locations. Therefore, it is necessary to adjust the dataset and decrease the bias on different

471 subsurface structures from the different number of samples, by selecting more high amplification

472 basins and reducing the number of low amplification hard rock sites in the dataset, for example.

473 Furthermore, detection of coherent peaks depends on the presence of representative samples from

474 closely related geological settings. This can be achieved by increasing data locations or utilizing

475 data from denser points over a smaller region.

\section{Conclusions and Discussions}

478 In this study, recurrent neural networks were utilized to predict site effects from earthquake coda

timeseries. In the first step, MSE values were relatively small on both training and test coda sets

on all site proxies. Although the scatter of predicted values against true values are somewhat large

on predictions of unique samples, it is much smaller on average for predictions at unique stations.

SD values in the case of Vs30 lie between $20 \mathrm{~m} / \mathrm{s}$ and $200 \mathrm{~m} / \mathrm{s}$ which is comparable with 

should be able to minimize this scatter.

486 The scatter occurs mainly due to the outlier data, degrading the network's performance. Since the 487 dataset is so large, it is crucial to deal with outliers and increase data quality as much as possible.

488 Among the many methods to tackle this issue, an RNN autoencoder was utilized in this study, to detect and rule out least correlated samples. Additionally, tuning network hyperparameters and optimizing data processing parameters could improve results which is yet to be done in this study.

491 A third reason causing scattered predictions is possibly the LSTM architecture. The conventional

492 LSTM networks are meant for classification tasks as opposed to regression. This means the

493 structure of memory cells is most suitable for outputs closer to 0 or 1 as possible, making it less

494 sensitive to uniformly distributed continuous values. Therefore, a more robust way to utilize

495 RNNs in a regression problem and achieve continuous value targets from sequential data is through a hybrid model. In a hybrid model, the RNN acts as a feature extraction algorithm and a separate regressor is used to infer the target value from those features. Lastly, as a general rule, adding more data samples helps improve the performance of machine learning models. In this 
In the second step, site amplification ratios were computed as surface to bedrock ratios of FAS of were able to extract the correct factors from the coda timeseries whereby to amplify bedrock ground motion. However, from investigating event and site characteristics of these inconsistent examples, it was evident that the RNN models were not able to account for directionality and topographical irregularities.

The future prospect of this study is to utilize easily obtainable microtremor timeseries instead of coda time-series and predict site effects at any arbitrary site in Japan. Both waves are believed to 
517 observations can be easily conducted anywhere, currently there is not enough observed data to

518 directly use for training a machine learning model. On the other hand, there is an abundant

519 earthquake dataset available, from which thousands of coda wave time windows can be extracted.

520 Intuitively, one can attempt to train RNN models on coda timeseries, and using the same models,

521 make predictions on microtremor timeseries. To investigate this idea, a few tentative tests were

522 run in both steps of the study. However, on the microtremor dataset, the MSEs were higher and

523 the predictions were as good as a random normal distribution with almost identical mean

524 prediction values and similarly large standard deviations for all stations. This indicates first and

525 foremost, that RNNs fail to simply treat microtremor and coda time windows as equivalents.

526 Although by visually inspecting the HVR plots at multiple stations, S-wave, coda wave and

527 microtremor wave seem to exhibit rather identical shapes, there are still differences, for example

528 in the exact peak frequencies and peak amplitudes. Considering the limited duration of earthquake

529 records, it is unlikely that any coda wave with no S-wave influence can be extracted. One solution

530 would be to minimize the S-wave to coda wave power ratio by utilizing lower PGA records.

531 Another solution would be to erase S-wave traces through deterministic procedures such as

532 procedures by Sánchez-Sesma et al. (2012) or Froment et al. (2011). Another imaginable 
approach is a simple additional step to map coda timeseries to their counterpart microtremor

534 timeseries. That is to say, if another sub model is trained to map between coda input and

535 microtremor input utilizing the 27 stations where both records are available, a larger network can

536 be trained on data from all KiK-net stations and make predictions from microtremor, by either

537 implementing the sub model prior to creating the training samples or prior to making predictions.

538 Another future prospect of the study is to tackle nonlinearity and directionality, which are the

539 most challenging aspects of evaluation of site effects. Although the current results are only a first

540 step in this direction, with proper datasets categorically sorted to, it should be possible to deal

541 with such challenging aspects in a similar manner.

542 The main takeaway from the study is that RNN algorithms can offer a fast and simple method of

543 extracting site specific information from scattered wavefield timeseries. In future, we expect to

544 use microtremor time-series in a similar manner and achieve reliable knowledge on the effect of

545 subsurface structure by non-invasive means.

546

547

548 
549 List of Abbreviations

550 Artificial Neural Network (ANN)

551 Coefficient of Variation (CV)

552 Earthquake Horizontal-to-Vertical (H/V) Spectral Ratios (EHVR)

553 Energy Ratio (ER)

554 Fourier Amplitude Spectra (FAS)

555 Kiban Kyoshin Network (KiK-net)

556 Long Short-Term Memory (LSTM)

557 Machine Learning (ML)

558 Mean Absolute Error (MAE)

559 Mean Squared Error (MSE)

560 National Research Institute for Earth Science and Disaster Resilience (NIED)

561 Peak Ground Acceleration (PGA)

562 Recurrent Neural Network (RNN)

563 Root Mean Squared Error (RMSE)

564 Standard Deviation (SD)

565 Surface to Bedrock Ratio (SBR)

566

567 


\section{Declarations}

569

570

571

572 The microtremor data will be shared on reasonable request to the corresponding author.

573 Competing interests

574 The authors declare that they have no competing interests.

\section{$575 \quad$ Funding}

576 Part of this study was supported by internal research funds of Disaster Prevention Research

577 Institute, Kyoto University.

578

Authors' contributions

579

M.I. and S.M. designed the study, M.I. processed the data and conducted the analysis and M.I.

580

and S.M. wrote the manuscript.

581

Acknowledgments

582

The authors pay regards to National Research Institute for Earth Science and Disaster Resilience

583

(NIED) for providing open access to strong motion records at KiK-net stations as well as J-SHIS 


\section{References}

592 Abadi, M., Agarwal, A., Barham, P., Brevdo, E., Chen, Z., Citro, C., Corrado, G.S., Davis, A.,

593 Dean, J., Devin, M. \& Ghemawat, S., 2016. Tensorflow: Large-scale machine learning on

594 heterogeneous distributed systems. arXiv preprint arXiv:1603.04467.

595 Aki, K., 1969. Analysis of the seismic coda of local earthquakes as scattered waves, J. Geophys.

596 Res., 74(2), 615-631.

597 Aoi, S., Kunugi, T. \& Fujiwara, H., 2004. Strong-motion seismograph network operated by

NIED: K-NET \& KiK-net, J. Japan Assoc. Earthq. Eng., 4(3), 65-74. 
DeVries, P.M., Viégas, F., Wattenberg, M. \& Meade, B.J., 2018. Deep learning of aftershock

Gupta, M., Gao, J., Aggarwal, C.C. \& Han, J., 2013. Outlier detection for temporal data: A survey.

IEEE Transactions on Knowledge and data Engineering, 26(9), pp.2250-2267.

Héloïse, C., Bard, P.-Y. \& Rodriguez-Marek, A., 2012. Site effect assessment using KiK-net data:

Part 1. A simple correction procedure for surface/downhole spectral ratios, Bull. Earthq. Eng.,

611 $10(2), 421-448$.

Hochreiter, S. \& Schmidhuber, J., 1997. Long short-term memory, Neural computation, 9(8),

614 Huang, J.P., Wang, X.A., Zhao, Y., Xin, C. \& Xiang, H., 2018. Large earthquake magnitude 615 prediction in Taiwan based on deep learning neural network. Neural Network World, 28(2), 
617 Iturrarán-Viveros, U., Muñoz-García, A.M., Parra, J.O. \& Tago, J., 2018. Validated artificial

618 neural networks in determining petrophysical properties: A case study from Colombia.

619 Interpretation, 6(4), pp.T1067-T1080.

620 Japan Seismic Hazard Information Station, 2012. NIED : National Research Institute for Earth

621 Science and Disaster Resilience, https://www.j-shis.bosai.go.jp/en/.

622 Kawase, H., Mori, Y. \& Nagashima, F., 2018. Difference of horizontal-to-vertical spectral ratios

623 of observed earthquakes and microtremors and its application to S-wave velocity inversion based

624 on the diffuse field concept, Earth, Planets and Space, 70(1).

625 Kieu, T., Yang, B. \& Jensen, C.S., 2018. Outlier detection for multidimensional time series using

626 deep neural networks, 19th IEEE Int. Conf. Mobile Data Management (MDM). IEEE.

627 Malhotra, P., Ramakrishnan, A., Anand, G., Vig, L., Agarwal, P. \& Shroff, G., 2016. LSTM-

628 based encoder-decoder for multi-sensor anomaly detection. arXiv preprint arXiv:1607.00148.

629 Margerin, L., Campillo, M., Van Tiggelen, B.A. \& Hennino, R., 2009. Energy partition of seismic

630 coda waves in layered media: theory and application to Pinyon Flats Observatory. Geophysical

631 Journal International, 177(2), pp.571-585. 
632 Matsuoka, M., Wakamatsu, K., Fujimoto, K. \& Midorikawa, S., 2005. Nationwide site 633 amplification zoning using GIS-based Japan Engineering Geomorphologic Classification Map,

634 Proc. 9th ICOSSAR, 239-246.

635 Mayeda, K. \& Walter, W.R., 1996. Moment, energy, stress drop, and source spectra of western

636 United States earthquakes from regional coda envelopes, J. Geophys. Res. Solid Earth, 101(B5),

$637 \quad 11195-11208$

638 Mignan, A. \& Broccardo, M., 2019. Neural Network Applications in Earthquake Prediction

639 (1994-2019): Meta-Analytic Insight on their Limitations, arXiv preprint arXiv:1910.01178.

640 National Research Institute for Earth Science and Disaster Resilience, 2019. NIED K-NET, KiK-

641 net, National Research Institute for Earth Science and Disaster Resilience, 642 doi:10.17598/NIED.0004.

643 Okada, Y., Kasahara, K., Hori, S., Obara, K., Sekiguchi, S., Fujiwara, H. \& Yamamoto, A., 2004.

644 Recent progress of seismic observation networks in Japan-Hi-net, F-net, K-NET and KiK-net-

645 Earth, Planets and Space, 56(8), pp.xv-xxviii. 
646 Raschka, S., \& Mirjalili, V., 2017. Python Machine Learning: Machine Learning and Deep

647 Learning with Python Scikit-Learn, and TensorFlow, 2nd ed Birmingham, UK: Packt Publishing 648 Ltd.

649 Rumelhart, D.E., Hinton, G.E. \& Williams, R.J., 1986. Learning representations by back650 propagating errors, Nature, 323(6088), 533-536.

651 Satoh, T., 2004. Study on envelope model of ground motions based on inversion of group delay

652 time and scattering theory, J. Struct. Constr. Eng., AIJ, 586, 71-78.

653 Sanchez-Sesma, F.J., Pina, J., Campillo, M., Luzón, F., García-Jerez, A., Albarello, D. \& Lunedei, 654 E., 2012, December. Seismic ambient noise H/V spectral ratio using the ACA (autocorrelations 655 of coda of autocorrelations) approach. In AGU Fall Meeting Abstracts (Vol. 2012, pp. S52C-04).

Satoh, T., Kawase, H. \& Matsushima, S., 2001. Differences between site characteristics obtained 
Table 1. RNN model parameters

\begin{tabular}{|c|c|c|}
\hline models & n_LSTM & activation \\
\hline A & 2 & tanh \\
\hline B & 3 & tanh \\
\hline C & 2 & sigmoid \\
\hline D & 3 & sigmoid \\
\hline
\end{tabular}

664

665 
667

\begin{tabular}{|c|c|c|c|c|c|c|c|}
\hline \multicolumn{2}{|l|}{ RMSE } & \multicolumn{2}{|c|}{$\mathrm{Vs} 30[\mathrm{~m} / \mathrm{s}]$} & \multicolumn{2}{|c|}{$\mathrm{Vs} 10[\mathrm{~m} / \mathrm{s}]$} & \multicolumn{2}{|c|}{$f_{0}[\mathrm{~Hz}]$} \\
\hline coda parameters & model & train & test & train & test & train & test \\
\hline \multirow{4}{*}{$(\mathrm{CV}=0.1, \mathrm{ER}=0.8)$} & A & 186.7 & 299.3 & 127.7 & 197.6 & 0.4 & 1.3 \\
\hline & B & 185.5 & 299.6 & 123.7 & 190.3 & 0.4 & 1.2 \\
\hline & $\mathrm{C}$ & 187.7 & 306.4 & 124.2 & 195.1 & 0.5 & 1.3 \\
\hline & $\mathrm{D}$ & 219.0 & 303.8 & 123.2 & 192.5 & 0.4 & 1.2 \\
\hline \multirow{4}{*}{$(\mathrm{CV}=0.2, \mathrm{ER}=0.9)$} & A & 187.2 & 296.8 & 123.1 & 191.7 & 0.4 & 1.3 \\
\hline & B & 184.2 & 301.8 & 159.4 & 190.2 & 0.4 & 1.3 \\
\hline & $\mathrm{C}$ & 186.0 & 307.6 & 124.9 & 192.6 & 0.4 & 1.3 \\
\hline & $\mathrm{D}$ & 188.7 & 296.0 & 129.6 & 194.2 & 1.1 & 1.2 \\
\hline
\end{tabular}

668

669 
Table 3. Prediction results for two cases for the EW component

\begin{tabular}{|c|c|c|c|c|}
\hline & Cs_test & Cs_train & Cc_test & Cc_train \\
\hline MAE & 4.31 & 3.32 & 3.78 & 3.39 \\
\hline SD & 4.93 & 7.80 & 7.67 & 7.50 \\
\hline
\end{tabular}

672

673 
674 Figure 1. Examples of EHVRs and selected predominant frequencies. Grey lines show the H/V

675 spectral ratios calculated for unique earthquake events, black lines show average EHVR of all

676 events recorded at the station and automatically detected first peaks are shown by orange circles.

677 Figure 2. H/V energy ratios for 2 sample events at MIEH005. a) sample event 1 and b) sample

678 event 2. Grey line indicates the threshold value $(=2)$.

679 Figure 3. Coda wave extraction based on the energy envelope for EW component of sample event

6801 of Fig. 2. a) Energy envelope with the extracted coda part in orange. b) Timeseries with the

681 extracted coda part in orange. Top: $\mathrm{C} 1\left(e r_{\text {thres }}=0.8, c v_{\text {thres }}=0.1\right)$, Bottom: $\mathrm{C} 2\left(e r_{\text {thres }}=0.9\right.$,

$682 c v_{\text {thres }}=0.2$.

683 Figure 4. Coda wave extraction based on the energy envelope for EW component of sample event

6842 of Fig. 2. a) Energy envelope with the extracted coda part in orange. b) Timeseries with the

extracted coda part in orange. Top: $\mathrm{C} 1\left(e r_{\text {thres }}=0.8, c v_{\text {thres }}=0.1\right)$, Bottom: $\mathrm{C} 2\left(e r_{\text {thres }}=0.9\right.$,

686

$\left.c v_{\text {thres }}=0.2\right)$.

687 Figure 5. Implemented RNN model for single output regression, model B parameters.

Figure 6. Implemented RNN autoencoder model for outlier detection. 
Figure 7. EW component of coda and microtremor records and reconstruction errors at 3 sample

stations. a) Coda with small MSE. b) Coda with large MSE. c) Microtremor. Original and red respectively. SD of calculated HVRs are shown in light shades. from training and plots. a) Vs30. b) Vs10. c) $f_{0}$. Light blue and dark blue dots show unique test sample results, and averaged results per station respectively.

Figure 10. Prediction errors per station for test set, sorted by difference of the two errors divided are shown in blue and orange respectively. 
Figure 11. Examples of fits of SBRs at stations with similar Cs and Cc errors (middle length bars on Fig. 10). a) EHMH10. b) FKOH07. c) HRSH01. d) GIFH25. e) TKCH07. f) NMRH05. Top :

705 Cs, Bottom: Cc.

706 Figure 12. Examples of fits of SBRs at stations with highest difference between Cc and Cs errors

707 (left hand bars on Fig. 10). Predicted peaks are more coherent in Cc. a) AICH06. b) FKOH04. c)

708 MYZH14. Top: Cs, Bottom: Cc.

709 Figure 13. Examples of fits of SBRs at stations with highest Cc error (right hand bars on Fig. 10).

710 a) ISKH05. b) MYGH01. c) TSKH02. Top: Cs, Bottom: Cc. Stations with highest Cc error also

711 tend to have similarly high Cs error.

712 Figure 14. Fits of predicted amplitudes of SBR in three frequency ranges. a) $f=1.0 \mathrm{~Hz}$. b) $f=5.0$ Hz. c) $f=9.0$ Hz. Top: Cs, Bottom: Cc. 
Figures
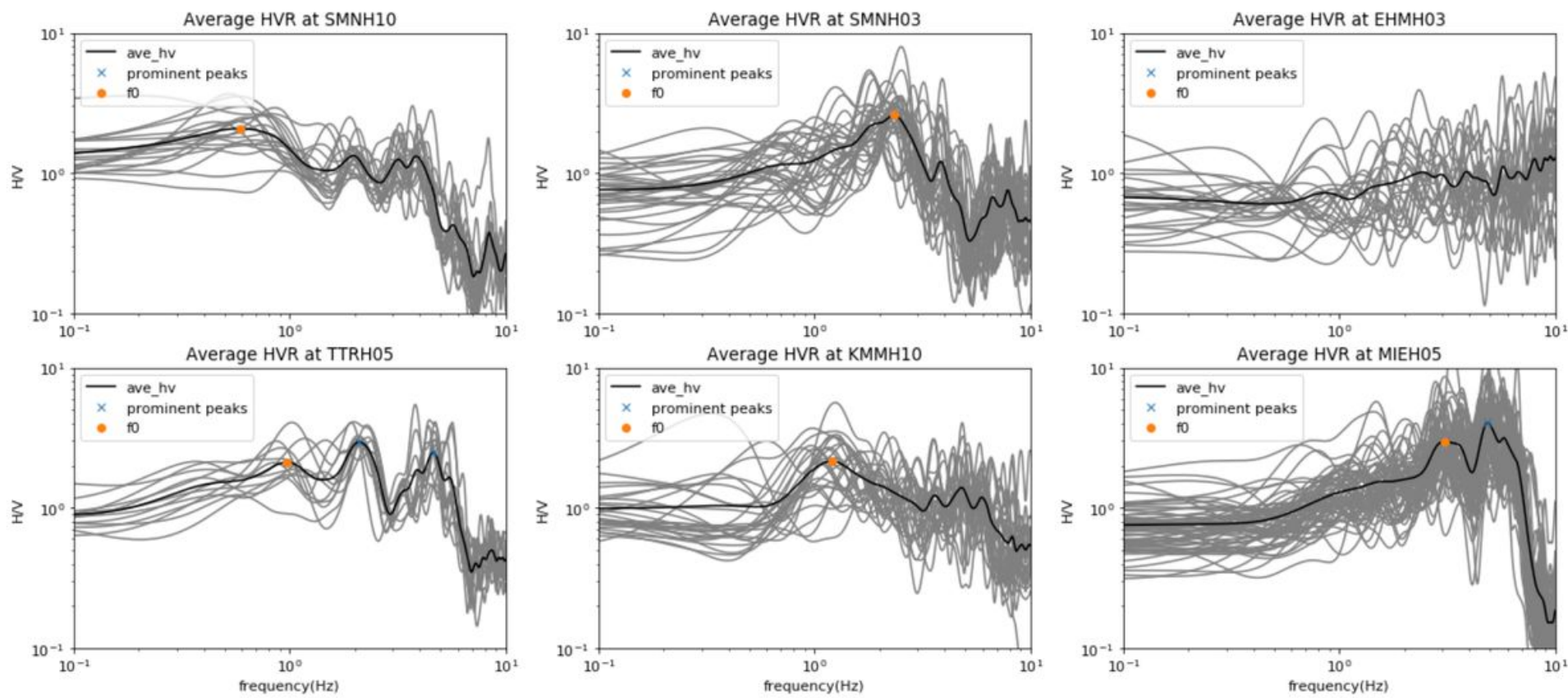

Figure 1

Examples of EHVRs and selected predominant frequencies. Grey lines show the H/V spectral ratios calculated for unique earthquake events, black lines show average EHVR of all events recorded at the station and automatically detected first peaks are shown by orange circles.
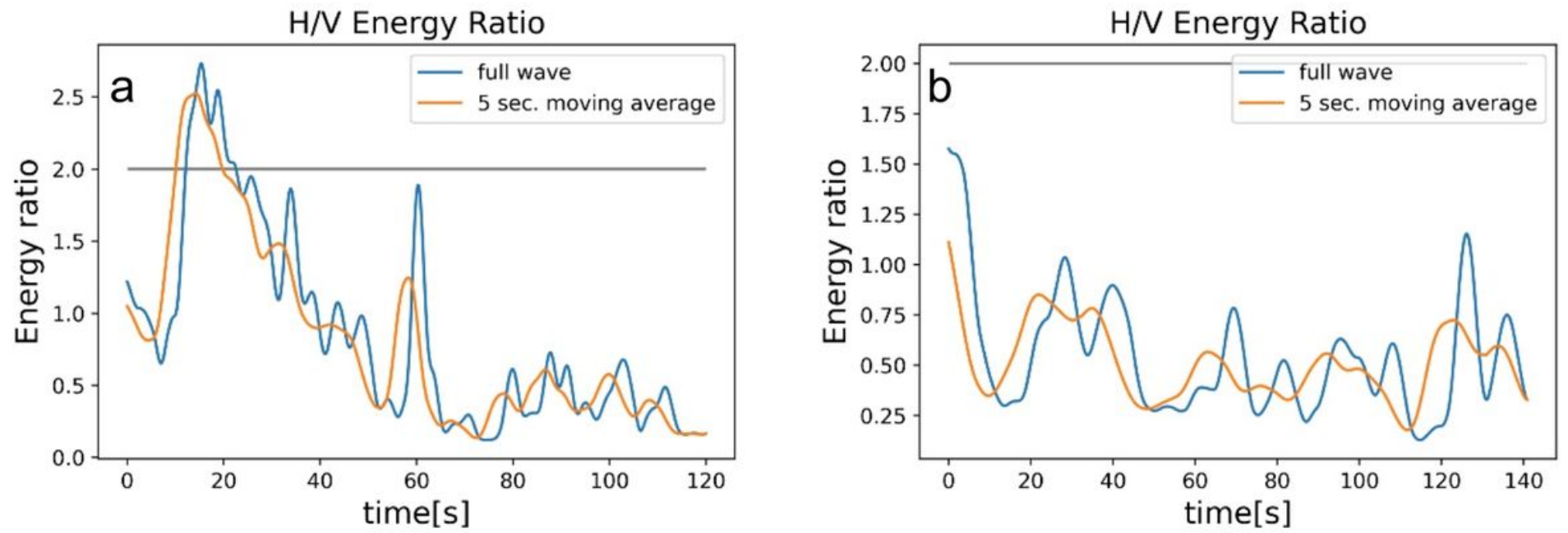

Figure 2

$\mathrm{H} / \mathrm{V}$ energy ratios for 2 sample events at MIEH005. a) sample event 1 and b) sample event 2. Grey line indicates the threshold value $(=2)$. 

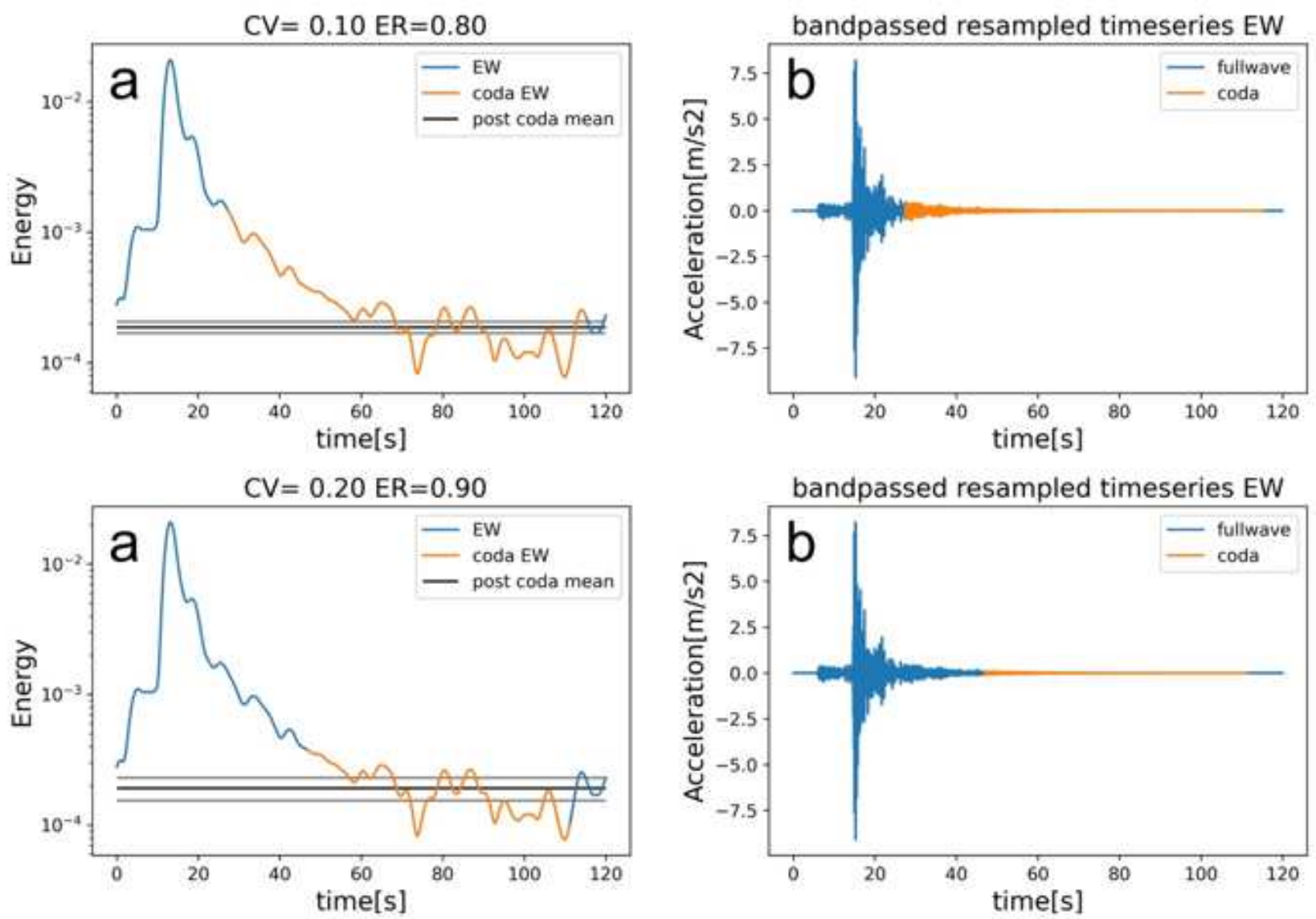

Figure 3. Coda wave extraction based on the energy envelope for EW component of sample event

1 of Fig. 2. a) Energy envelope with the extracted coda part in orange. b) Timeseries with the extracted coda part in orange. Top: $\mathrm{C} 1\left(e r_{\text {thres }}=0.8, c v_{\text {thres }}=0.1\right)$, Bottom: $\mathrm{C} 2\left(e r_{\text {thres }}=0.9\right.$, $\left.c v_{\text {thres }}=0.2\right)$.

\section{Figure 3}

See image above for figure legend 

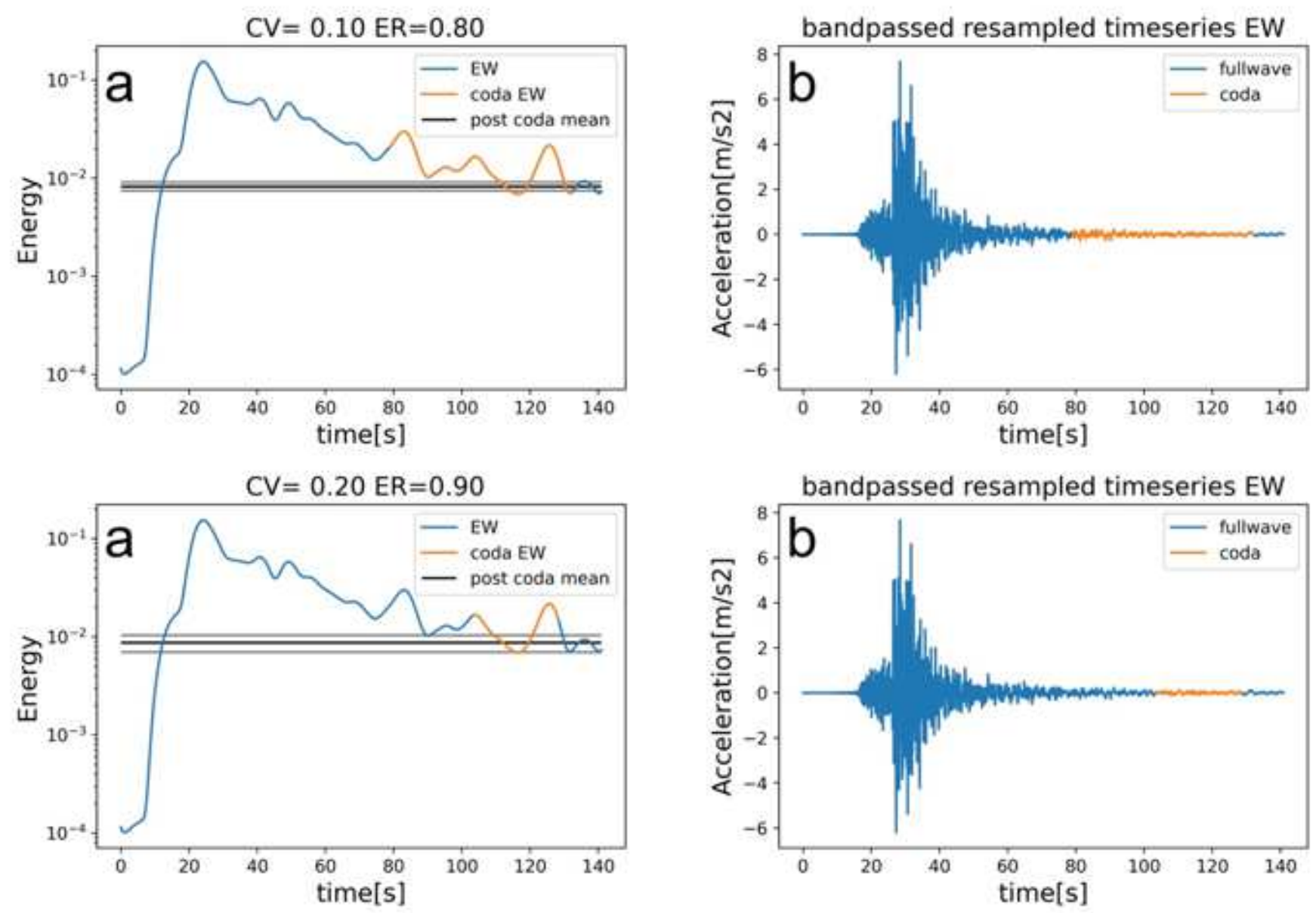

Figure 4. Coda wave extraction based on the energy envelope for EW component of sample event 2 of Fig. 2. a) Energy envelope with the extracted coda part in orange. b) Timeseries with the extracted coda part in orange. Top: $\mathrm{C} 1\left(e r_{\text {thres }}=0.8, c v_{\text {thres }}=0.1\right)$, Bottom: $\mathrm{C} 2\left(e r_{\text {thres }}=0.9\right.$, $c v_{\text {thres }}=0.2$ ).

\section{Figure 4}

See image above for figure legend 
Layer input: $n_{s} \times 3 \times 100$

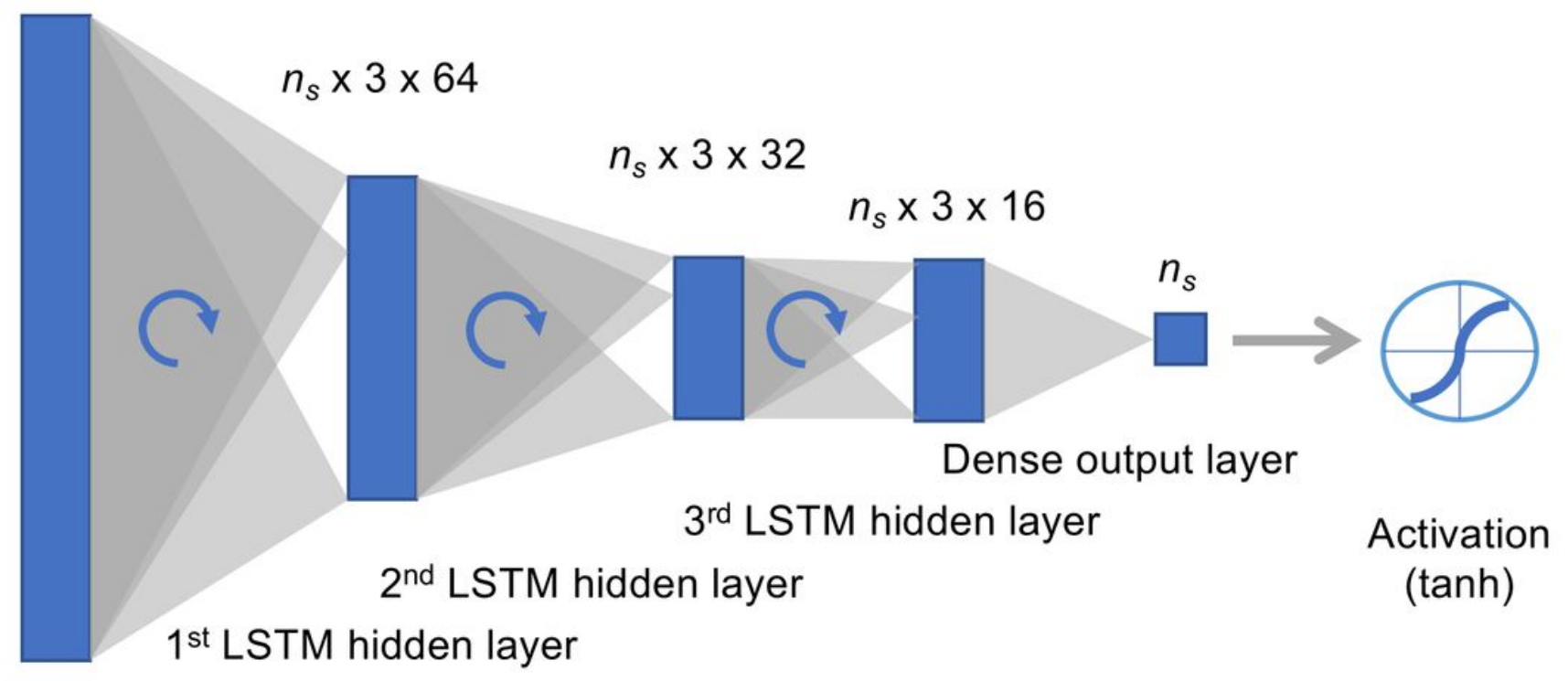

Figure 5

Implemented RNN model for single output regression, model B parameters. 
Layer input: $1 \times n_{t}$

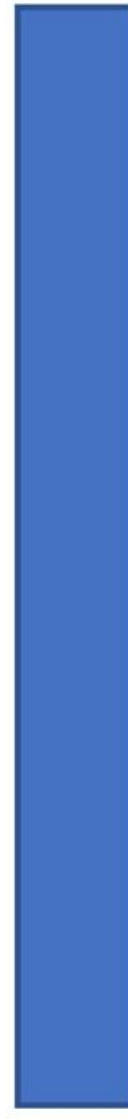

$1^{\text {st }}$ LSTM hidden layer

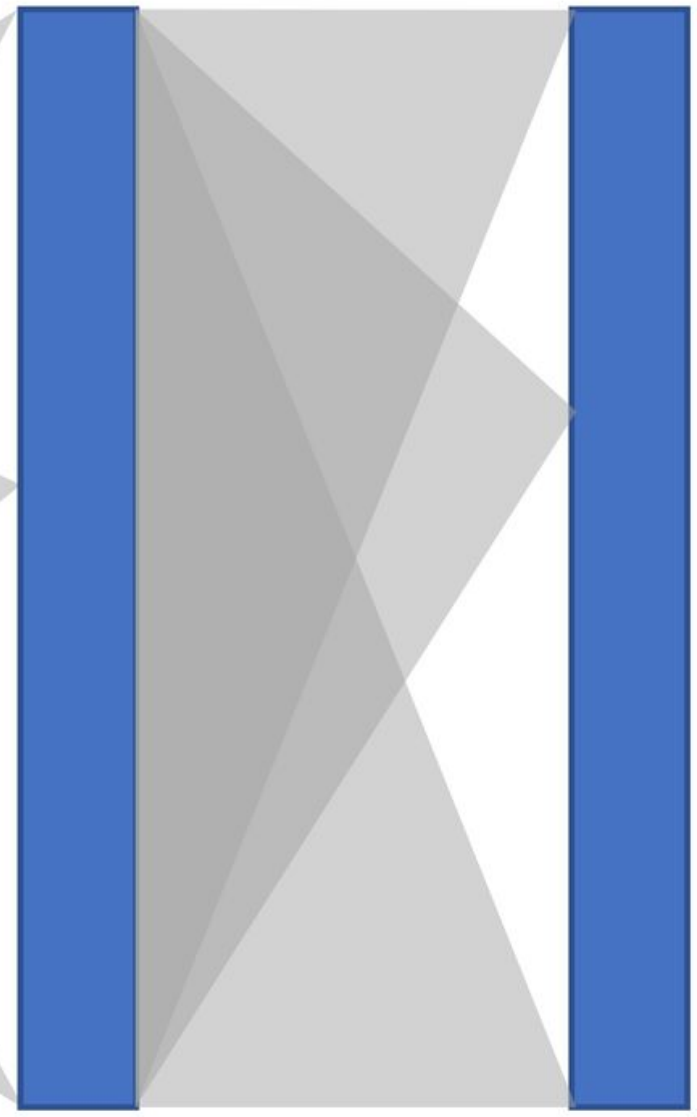

Dense output layer (encoder)

$2^{\text {nd }}$ LSTM hidden layer

(decoder)

Figure 6

Implemented RNN autoencoder model for outlier detection. 

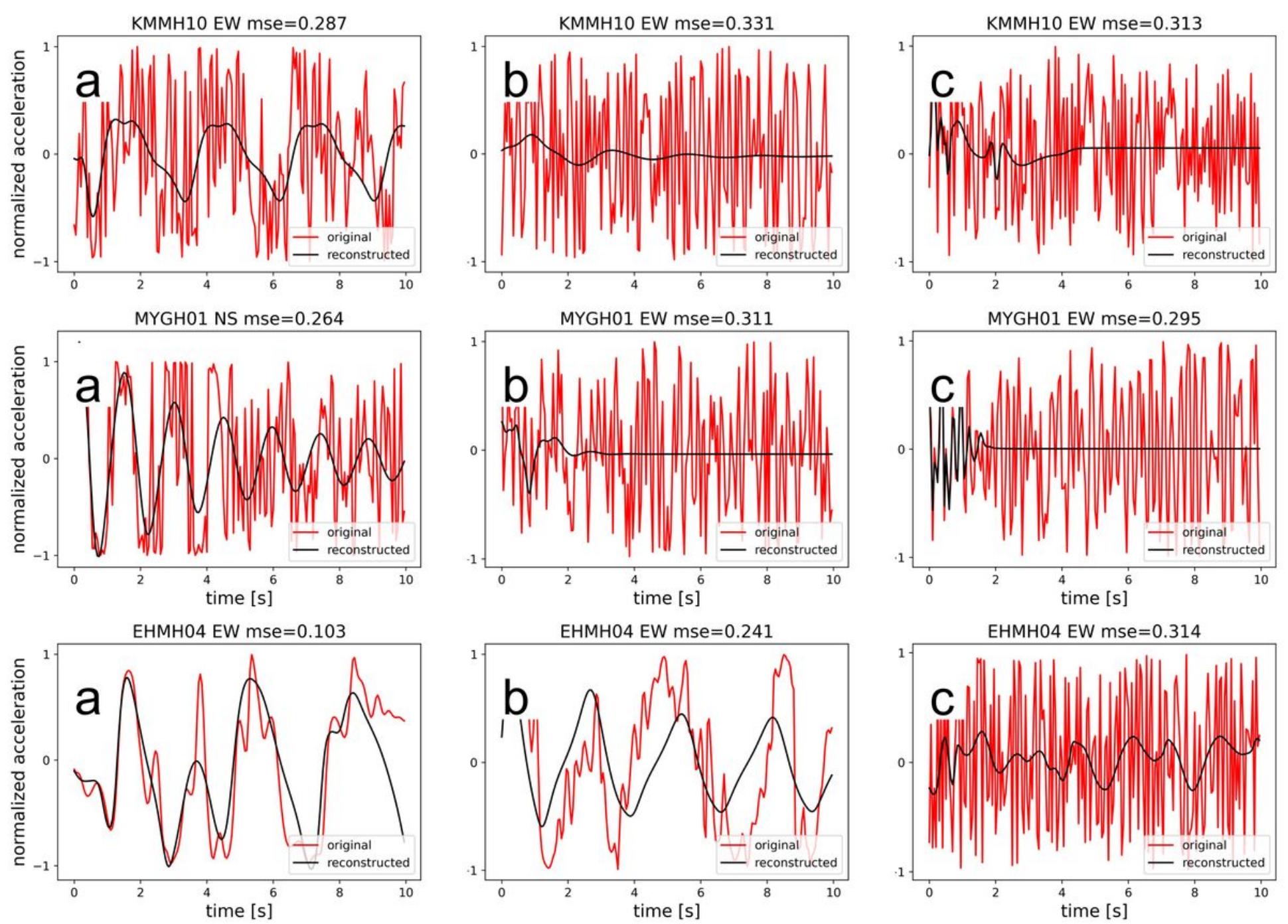

Figure 7

EW component of coda and microtremor records and reconstruction errors at 3 sample stations. a) Coda with small MSE. b) Coda with large MSE. c) Microtremor. Original and reconstructed signals are shown in red and black respectively. Top: KMMH10 station, Middle: MYGH01 station, Bottom: EHMH04 station.
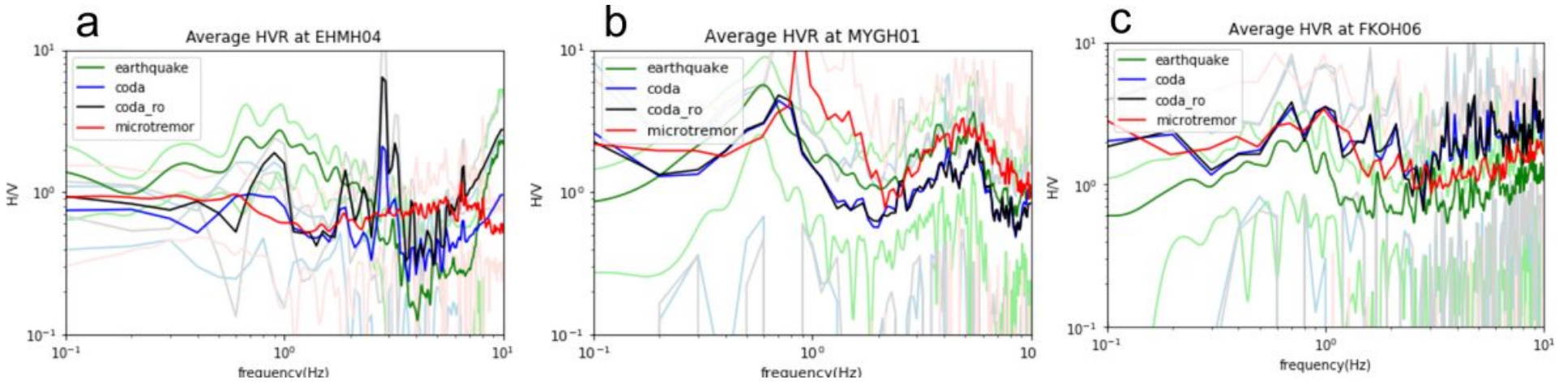

Figure 8

Examples of HVR of earthquakes, coda, and microtremors at 3 sample stations. a) EHMH04 station. b) MYGH01 station. c)FKOH06 station. Average HVR of earthquakes, coda including outliers, coda without 
outliers and microtremors are shown in green, blue, black and red respectively. SD of calculated HVRs are shown in light shades.
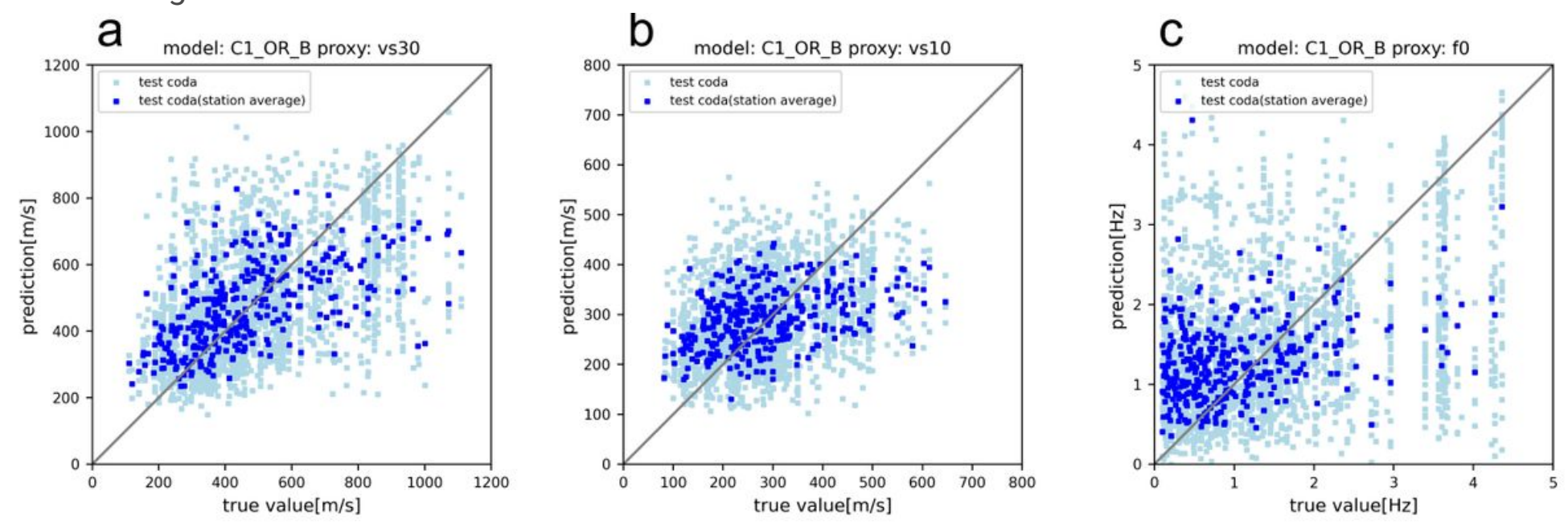

Figure 9

Prediction results on test set, for model $\mathrm{B}$ trained on $\mathrm{C} 1$ dataset with $\mathrm{z}$-score $>3$ excluded from training and plots. a) Vs30. b) Vs10. c) f0. Light blue and dark blue dots show unique test sample results, and averaged results per station respectively.

$100-a$

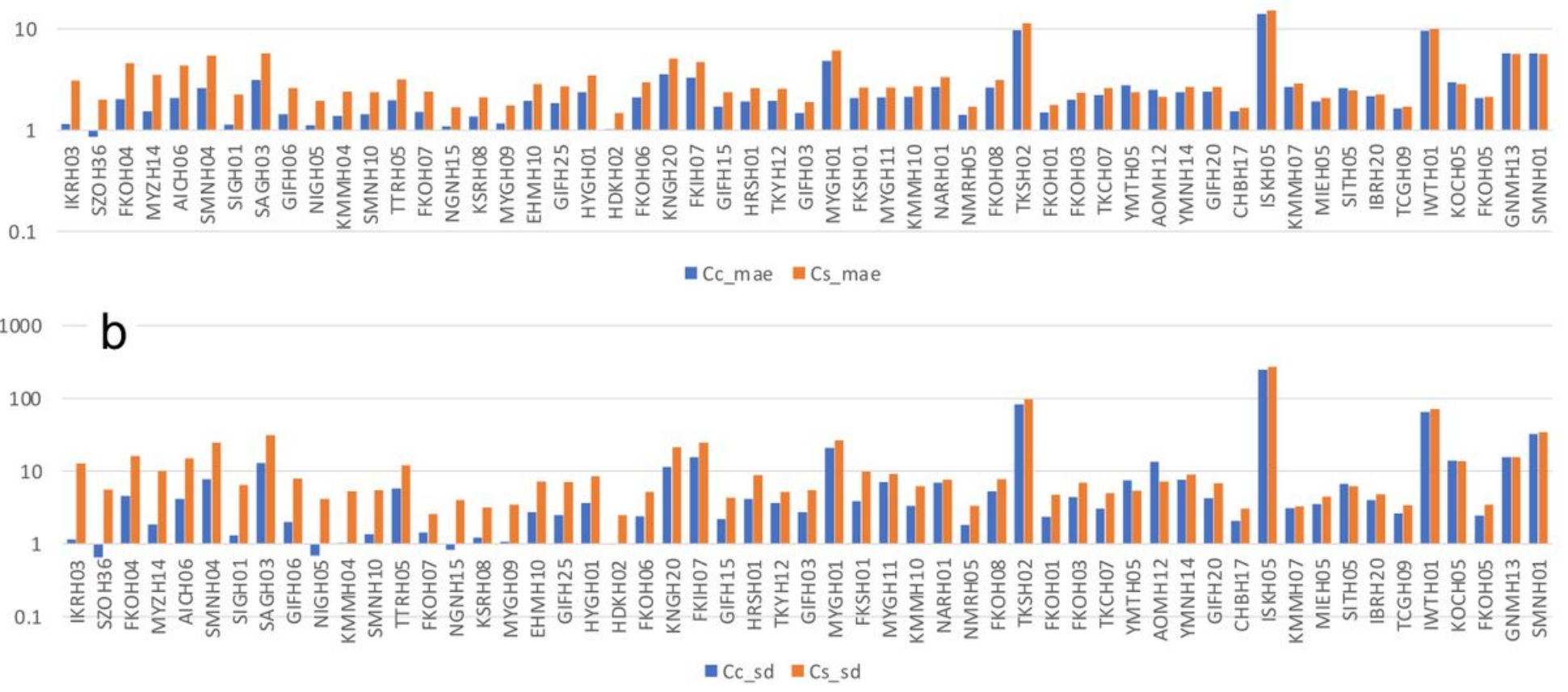

Figure 10

Prediction errors per station for test set, sorted by difference of the two errors divided by Cs error in decreasing order from left to right. a) MAE. b) SD. Error bars for Cc and Cs cases are shown in blue and orange respectively. 

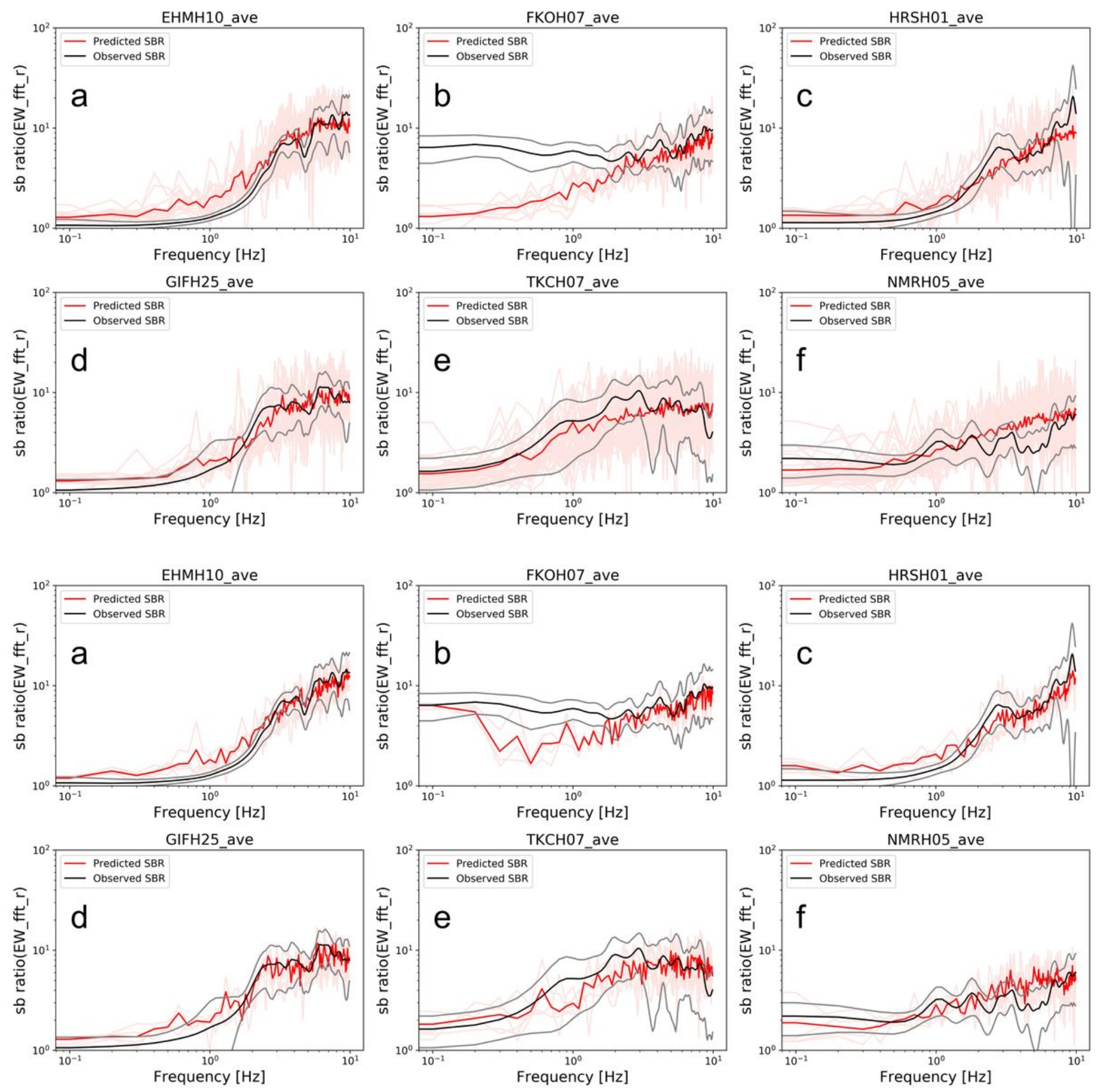

\section{Figure 11}

Examples of fits of SBRs at stations with similar Cs and Cc errors (middle length bars on Fig. 10). a) EHMH10. b) FKOH07. c) HRSH01. d) GIFH25. e) TKCH07. f) NMRH05. Top囚Cs, Bottom: Cc. 

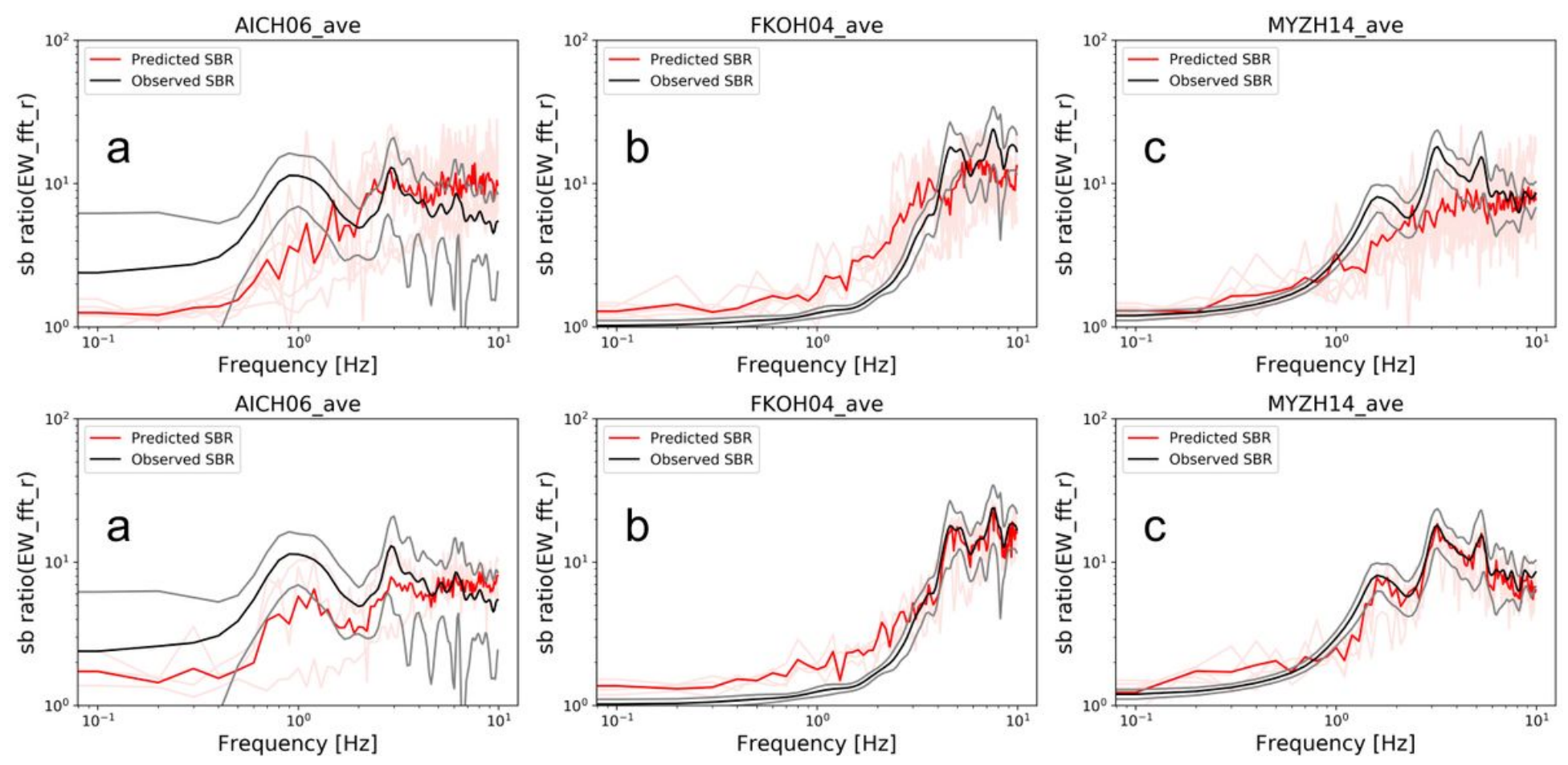

Figure 12

Examples of fits of SBRs at stations with highest difference between $\mathrm{Cc}$ and $\mathrm{Cs}$ errors (left hand bars on Fig. 10). Predicted peaks are more coherent in Cc. a) AlCH06. b) FKOHO4 . c) MYZH14. Top:Cs, Bottom: Cc.
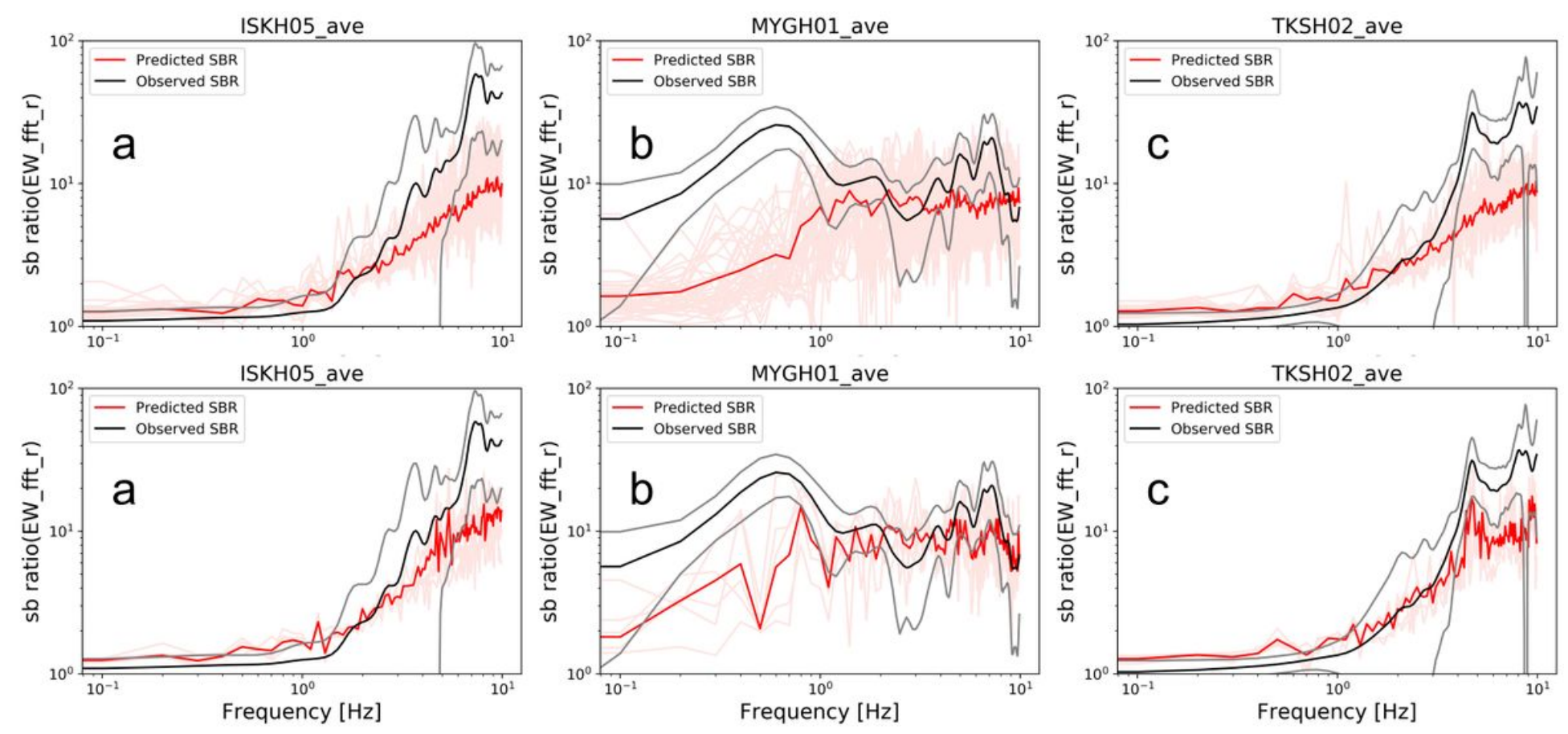

\section{Figure 13}

Examples of fits of SBRs at stations with highest Cc error also tend to have similarly high Cs error (right hand bars on Fig. 10). a) ISKH05. b) MYGH01. c) TSKH02. Top:Cs, Bottom: Cc. Stations with highest Cc 
error also tend to have similarly high Cs error.
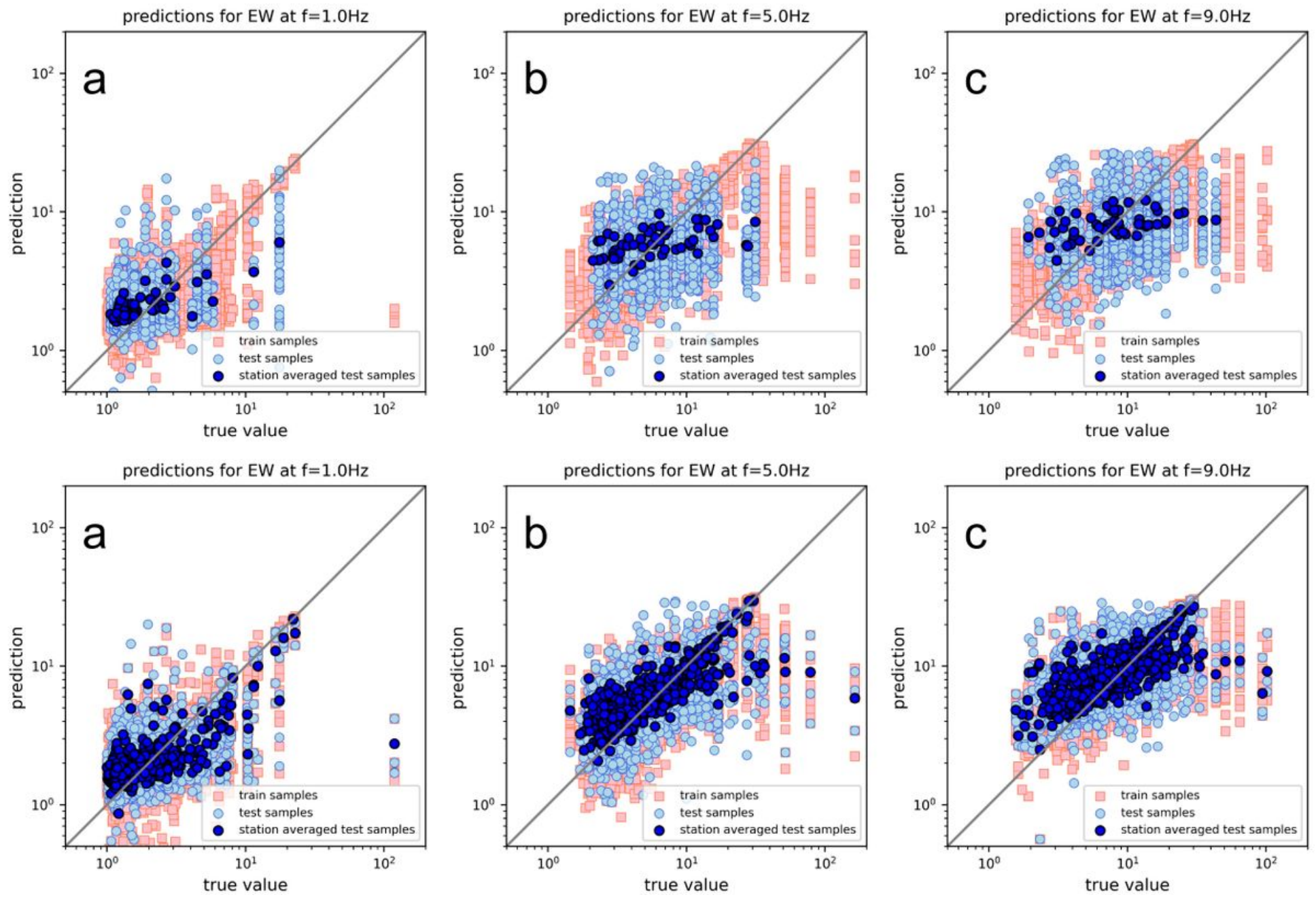

Figure 14

Fits of predicted amplitudes of SBR in three frequency ranges. a) $f=1.0 \mathrm{~Hz} . \mathrm{b}) \mathrm{f}=5.0 \mathrm{~Hz}$. c) $\mathrm{f}=9.0 \mathrm{~Hz}$. Top:Cs, Bottom: Cc.

\section{Supplementary Files}

This is a list of supplementary files associated with this preprint. Click to download.

- GraphicalAbstract.jpg 